hæmorrhage beyond control. By attacking the tonsil from without, we have no inconvenience to fear after the primary dangers of the operation, except the risk of a pharyngeal fistula.

Our cases of œsophagotomy, however, have demonstrated that openings into the gullet close readily. The case above reported closed earlier than the wounds of the œsophagus, partly because the opening was higher up, and partly because the pillars of the palate closed the fistula by a valve-like action of their own.

The facility with which the tonsil can be enucleated with the finger is surprising. The following anatomical peculiarity, however, explains the reason pretty well:

"Perhaps the most important point of the anatomy of the tonsils, next to their proximity to the internal carotid artery, is the fibrous semi-capsule described by Chassaignac, as follows: 'When one has enucleated a well developed tonsil, and examined with attention its external and internal surface, he does not hesitate to declare that the external, or adherent face, is covered with a fibrous semi-capsule, well circumscribed, independent of the neighboring aponeurosis, and resting on the cellular tissue.'

"The arteries of the tonsil are large compared with the size of the gland; they come from the ascending pharyngeal and the inferior and superior palatine arteries."

We desire, in the report of this interesting case, to express our appreciation of the aid we have received, in the history of the disease and other points, from Mr. F. W. Draper, House-surgeow, and Mr. C. B. Brigham.

\section{MASSACHUSETTS CHARITABLE EYE AND EAR INFIRMARY.}

Some Cases in the Service of Dr. B. Joy JeFr RIEs.

Congenital Cataract, Double.-A woman, aged 24, has congenital cataract, nystagmus and convergent strabismus. Vision enough to go about where acquainted. Oct. 30, 1867, iridectomy downwards on right, and, Nov. 12,1867, same on left, preparatory to extraction. By these, vision not much improved, and in reference to optic nerve atrophy there was a doubt as to extraction; however, five months afterwards, a corneal cut was made with iridectomy-knife in left eye, and with forceps capsule torn away, and cretaceous mass size of four pins' heads from centre of pupil. Patient suffered from abscess on finger, \&c., and absorption kept up consideVol. III.-No. $4 \mathrm{~A}$ rable irritation; yet in two weeks patient fixed with this eye, although there was still some débris of lens and capsule. April 13, 1868 , patient counts fingers and goes about readily, greatly gratified with improvement of sight. May 14th, 1868, same operation done on right eye. A little vitreous presented, but pupil was cleared. Patient had considerable pain, yielding to morphine. By May 31, 1868, can bear light; pupil quite clear. June 5, 1868, with $+2 \frac{1}{2}$ reads Jäger 14, and with $+\frac{1}{5}$ tells fingers at 7 feet. Both optic papillæ and fundus oculi now seen. The papillæ are irregular, as also retinal vascular distribution; no apparent disease. Oct. 14, 1868, patient reads Jäger 14 with any glass from $+\frac{1}{3}$ to $+\frac{1}{4}$, and makes out Dyer's X L at 20 feet. The absence of a white cataract filling the pupils, the steadiness of the globes and much improved vision greatly alter the appearance and comfort of this patient. The case is very instructive as to what we may have behind a congenital cataract, and how much even a by no means normal retina and nerve may serve to convey impressions to the brain. The distinction of color was good, the patient having won a new bonnet by selecting it, after operation. A brother and cousin also have congenital cataract.

Enucleation.-A domestic, aged 21, has large staphyloma of left eye, at junction of cornea and sclera. Some pain and sympathetic irritation of other eye. Rupture of staphyloma threatening. Patient desires enucleation for cosmetic effect, and also on account of trouble of other eye, "weakness." Oct. 30, 1867, globe enucleated. On section, lens clear; old pannus of cornea; choroid pale, and too readily separable from sclerotic; vitreous clear; staphyloma thin, and bulging irregularly. Vision had long been gone in this eye. Two hours after operation patient had no pain, and wanted food. Patient discharged on fifth day. One month later, patient came wearing false eye, with very perfect deception and considerable movement.

Cut through Cornea.-A boy, aged 12, in passing a mill hand, has one third of cornea cut through by end of a piece of wire the workman was pulling from a machine. Scratch on face shows direction of cut. Patient applied to a physician, who said " sight was running out," and applied caustic. Now, Nov. 11, 1867, great pain and ciliary redness. Pupil, irregularly dilated with atropine, showed synechia posterior and traumatic cataract. Leeches, atropine and rest abated symptoms, and 
- patient was discharged, Nov. 22d, with synechia posterior and traumatic cataract not then in condition to remove. It seemed almost impossible that the end of a wire could cut open the cornea so cleanly.

Iridectomy.-A man, aged 24, pretty well run down from graduation at rebel prisons, has opacity of two thirds of cornea of right eye. Constant pain and some sympathetic irritation; for this iridectomy was done inwards. Iris rotten, and removed all possible. Lens seen opaque and soft. Punctured, and flowed off the consistency of pus, and of sago-like color. This, March 28,1868 ; no subsequent pain, and eye "never so quiet." April 1, patient going about, and can see window. April 4, patient discharged. The whole probably sequelæ of ulcers of cornea and irido-choroiditis from starvation and exposure in Southern prison stockades.

Distichiasis Operation.-A woman, æt. 23, has distichiasis of both upper lids and ulcer of right cornea. Patient in poor condition, but improved by treatment, and ulcer healed. In two weeks, right upper lid "scalped," and two bunches of hair dissected from left upper lid. April 23, 1868, patient discharged, well. These eyes would have finally been destroyed, as many others have, from lack of this simple and very effective operation.

Cataract.-A man, aged 79 , has double cataract. Some debility, rheumatism in back, troublesome right inguinal hernia. April 14, 1868, left lens extracted by Græfe's method. Lens large and hard; some cortical removed with Daviel's spoon. Discharged, May 5, 1868, with pupil clearing. Sept. 22, 1868, patient returned for glasses. $\nabla=\frac{1}{5}$ at 20 feet, or 10 feet with $+\frac{1}{4}$. Reads Jäger 6 at 7 inches with $+\frac{1}{3}$. $+\frac{1}{3}$ ordered. Success better than anticipated in so old and weak a person.

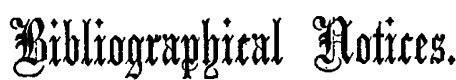

A New Operation for Artificial Hip-joint in Bony Anchylosis, illustrated by two Cases. By Lewis A. SAYRe, M.D., Surgeon to Bellevue Ilospital, Professor of Orthopædic Surgery, \&c. \&c. Re-printed, \&c. Pamphlet. 8vo. Pp. 39. New York: D. Appleton and Co. 1869.

These cases are re-published from the New York Medical Journal for January, 1869 , where they are re-printed from the Transactions of the Medical Society of the
State of New York, with Preface and Appendix, says Dr. Sayre, " to vindicate scientific truth, and my own reputation" from "false statements" with regard to them in a "work" recently published.

The vindication is thorough and complete-we need not tarry to rehearse the process-the "slander" henceforth can trouble only its inventor.

As these operations are remarkable, and "the first of their kind," the description, which is not long, is worthy of repetition.

"My object was to go above the trochanter minor so as to retain the insertion

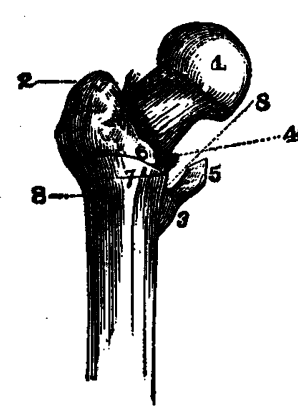
of the psoas magnus and the iliacus internus muscles attached to the lower fragment, for the purpose of flexion; and by cutting out a semi-circularpiece, thus $\frown$, with its convexity downward, and then rounding off the upper end of the lower section to more nearly imitate 1 , head of femur; 2 , tro- the natural .joint, and chanter major, trochan- give him a fair chance tion of capsular ligament for motion at that (variable); 5, tendon of point." The plan of psoas magnus and iliacus the operation may be internus muscle; 6, line of the peen in the figure. dotted lines indicating the This operation, derounding off of lower frag- signed by $\mathrm{Dr}$. Sayre ment after removal of seg- "for the purpose of
ment. establishing a new joint," was first performed on a man at the Bellevue Hospital, June 11th, 1862. The details and history are given with sufficient fulness in the pamphlet. October 12th he "left the institution well, and with very good motion " in the new joint. Late in December he walked to Dr. Sayre's office, and could go up and down the steps without difficulty. April 11 th, 1863, the patient wrote from Kentucky "I can now ' rough it' a little with. out apprehension of having to suffer from it afterward. I can bear my whole weight on the limb without inconvenience, and can walk very well without other assistance than a walking stick." (Page 13.) When last heard of, Jan. 9th, 1869, he was in Idaho, writing to New York for a twowheeled velocipede!

On the 6th of Nov., 1862, assisted by Profs. Peaslee and Raphael, and in presence of other professional gentlemen, Dr. Sayre performed a similar operation on a Miss $L$.

This case went on well so far as the operation was concerned. Its subsequent fa- 